\title{
Ein Arachniden-Opisthosoma aus dem Obervisé von NW-Sachsen
}

\author{
Eberhard Kahlert $^{1}$
}

Mit 1 Tafel und 2 Abbildungen

\section{Zusammenfassung}

Ein aus dem Unterkarbon von NW-Sachsen stammendes Opisthosoma wird als Cryptomartus sp. beschrieben. Damit liegt stratigraphisch nach bisherigen Erkenntnissen der älteste Fund dieses Genus vor.

Schlüsselwörter: Arachnida, Cryptomartus sp., Opisthosoma, Bohrprobe, Obervisé

\begin{abstract}
An Arachnid-Opisthosoma from the Lower Carboniferous of North Western Saxony

An opisthosoma of Cryptomartus sp. is described from the Lower Carboniferous of North Western Saxony. That this my knowledge is the oldest record for the genus.
\end{abstract}

Key words: Arachnid, Cryptomarus sp., opisthomsoma, drill test, Lower Carboniferous, NW-Saxony

\section{Einleitung}

Seit 1835 (Corda) wurden mehr oder weniger gut erhaltene Exemplare bzw. Reste fossiler Arachniden vorwiegend im geologischen Schrifttum beschrieben. Die bereits beträchtlich angewachsene Zahl der Fossilfunde aus dem Paläozoikum (mehr als 100 Gattungen in ca. 175 Arten) charakterisiert die Vielgestaltigkeit dieser Tiergruppe und läßt sie evolutionsmorphologisch als eine der erfolgreichsten erscheinen. In adaptiogenetischer Hinsicht ist die stammesgeschichtliche Entwicklung dieses Taxons durchaus nachvollziehbar. Strittig dagegen ist nach wie vor sein phylogenetischer Ursprung. Fraglich sind nicht nur die $\mathrm{Ab}$ grenzungen der einzelnen Genera der Spinnentiere, sondern auch die Hypothese, ob es sich bei ihnen um eine monophyletische Gruppe handeln könnte. Bei fossilen Arachniden gibt es z. Z. kaum hinreichend diagnosti-zierbare anatomische Einzelheiten, um in Anwendung der zoologischen Systematik überzeugende Cladogramme zu entwickeln (Hennig 1969). Für absehbare Zeit wird die von Petrunkevitch (1955) eingeführte Klassifikation die Grundlage für die taxonomische Bewertung fossiler Arachnida bleiben.

\section{Herkunft des Materials}

Der Arachniden-Fund befand sich in einer pelitischen Abfolge der Kartierungsbohrung Delitzsch $9 / 64$ bei einer Teufe von $231 \mathrm{~m}$ (Abb. 1). Aus diesem Bereich stammen auch die Florenfunde, die zur stratigraphischen Einstufung der Schichtenfolge in das Obervisé (evtl. striatus-Zone IIIß) führte (Kahlert 1975). Damit kommt diesem Fund ein besonderes Interesse $\mathrm{zu}$, da bisher aus diesem stratigraphischen Bereich kein Nachweis bekannt war.

Die organischen Substanzen des Fossilmaterials sind hoch inkohlt (Anthrazit-Stadium) und tragen z. T. einen Gümbelitbelag. Das Fundstück ist im Museum für Naturkunde der Humboldt-Universität zu Berlin unter der Nr. MB.A. 854 a, b hinterlegt.

\section{Systematik}

Klasse Arachnida Lamarck, 1801

U.Klasse Stethostomata Petrunkevitch, 1949

Ordnung Trigonotarbida Petrunkevitch, 1949

Familie Anthracomartidae Haase, 1890

Gattung Crytomartus Petrunkevitch, 1945

Typusart: Cryptomartus hindi Pocock, 1911

Beschreibung: Auf einem schwach siltigen Schieferton-Bohrkernstück liegt der dorsale Abdruck (mit Gegendruck) eines Opisthosoma ohne Prosoma, Pedipalpi und Extremitäten.

Der Umriß des Opisthosoma ist länglich-oval und deutlich in 7 Segmente gegliedert. Das erste

\footnotetext{
1 Museum für Naturkunde, Institut für Paläontologie, Invalidenstraße 43, D-10115 Berlin, Germany. Erhalten März 1998, angenommen Juni 1998
} 


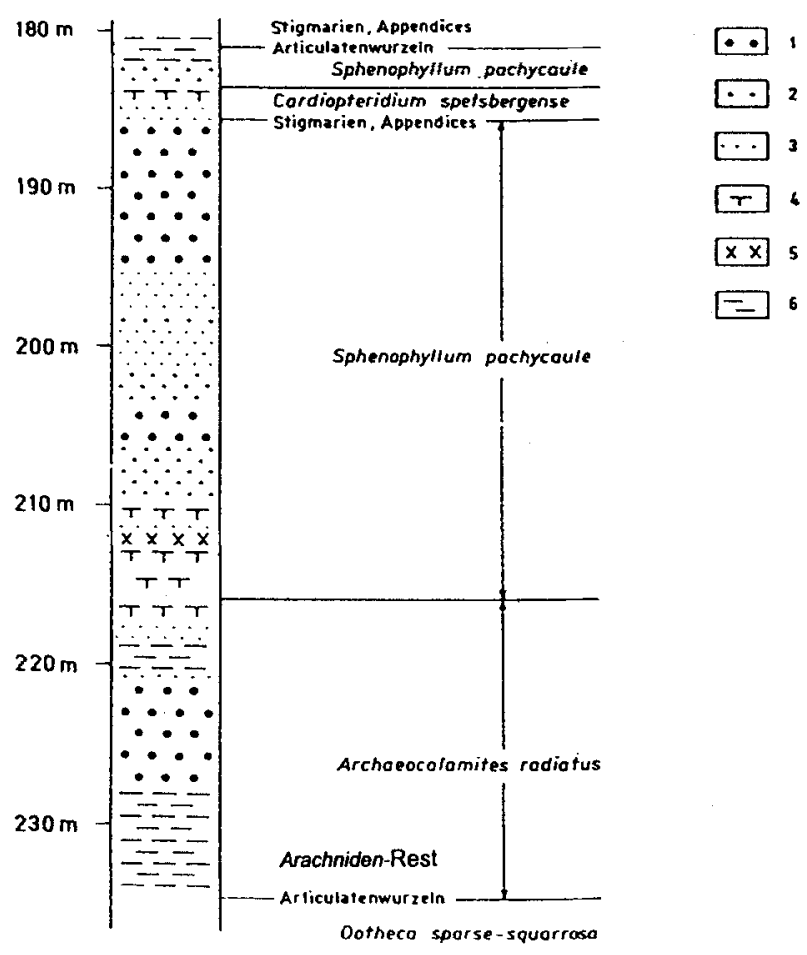

Abb. 1. Profilausschnitt der Bohrung Delitzseh 9/64 mit paläobotanischen Faziesbereichen. 1 - Grobsandstein; 2 - Mittelsandstein; 3 - Feinsandstein; 4 - Siltstein; 5 - Vulkanit; $\mathbf{6}$ - Tonstein

Fig. 1. Section from the core drilling Delitzsch $9 / 64$ with paleobotanical facies. 1 - Coarse sandstone; 2 - Middle sandstone; $\mathbf{3}$ - Fine sandstone; 4 - Siltstone; 5 - Vulcan rock; 6 - Argillit

Segment ist sehr breit angelegt. Die Oberfläche ist mehr oder weniger fein granuliert. Die Quergliederung des Dorsal-Schildes ist wie bei allen Anthracomartidae-Formen durch zwei Paar Longitudinal-Linien in eine mediane sowie beiderseits in je eine submarginale und eine marginale

\section{Tabelle 1}

Maße des Opisthosoma.

Table 1

Measurements of the opisthosoma.

\begin{tabular}{lr}
\hline Länge des Opisthosoma & $10,0 \mathrm{~mm}$ \\
Breite des Opisthosoma & $8,5 \mathrm{~mm}$ \\
Breite des Lateralsaumes & $1,0 \mathrm{~mm}$ \\
Somiten & $1,0 \mathrm{~mm}$
\end{tabular}

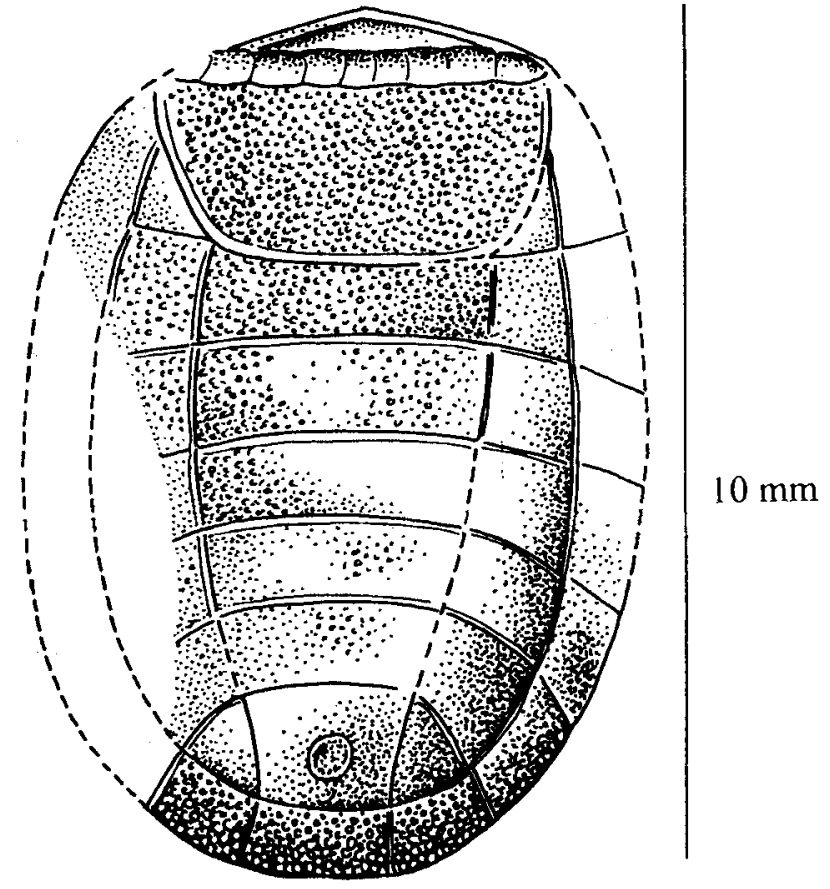

Abb. 2. Cryptomartus sp. Dorsalseite, von der Ventralseite das Anal-Operculum durchgedrückt

Fig, 2. Cryptomartus sp. Dorsal side, the arachnid is seen from the dorsal side, but an imprint of the ventral side is visible on the dorsal surface

Plattenreihe gegliedert. Von der Ventral-Seite aus ist das Anal-Operculum durchgeprägt (Abb. 2 u. Taf.).

Bemerkungen: In einer Reihe von Arbeiten von Brauckmann $(1984,1985,1987)$ werden paläozoische Arachniden-Funde hinsichtlich ihrer Stratigraphie, ihres regionalen Auftretens sowie sie betreffende Nomenklaturfragen diskutiert. Bezüglich seiner Aussage zur Priorität des Artnamens hoffmani wäre anzumerken, daß diese Architarbus hoffmani Guthörl (1934) zukommt. Die aus Ostdeutschland von Nindel (1955) und Simon (1971) publizierten Taxa sind unberücksichtigt geblieben. Weitere Arbeiten über Arachniden erschienen zudem von Daber $(1990,1992)$ und Rößler \& Schneider (1997).

Der Autor hält es für notwendig, in dieser Arbeit einige korrektive Anmerkungen über die Arbeit von Nindel (1955) zu implizieren.

Tafel.

Cryptomartus sp. 1 Opisthosoma, Dorsalseite, Abdruck $\times 5$, MB. A. $854 a ; 2$ Gegendruck $\times 5$, MB. A. 854 b; 3 Gegendruck, deutliche Tuberkulierung, Anal-Operculum von Ventralseite durchgedrückt, durch Longitudinal-Suturen bedingte Felderung in mediane und marginale Tergite $\times 11 ; 4$ Vergrößerter Ausschnitt von Fig. 3 des ersten Segmentes mit nicht deutbarer Querwulst (fossilisationsbedingt?), wulstartige Segmentgrenzen $\times 20$

Plate.

Cryptomartus sp. 1 Opisthosoma, dorsal side, as imprint $\times 5 ; 2$ Counterpart $\times 5 ; 3$ Counterpart with prominent tuberculation, anal operculum as imprint from ventral view; articulation in median and marginal tergits by longitudinal sutures $\times 11 ; 4$ Sector from fig. 3 first segment with no explicable swelling (? by fossilization), thick segment-limits 
Tafel 1

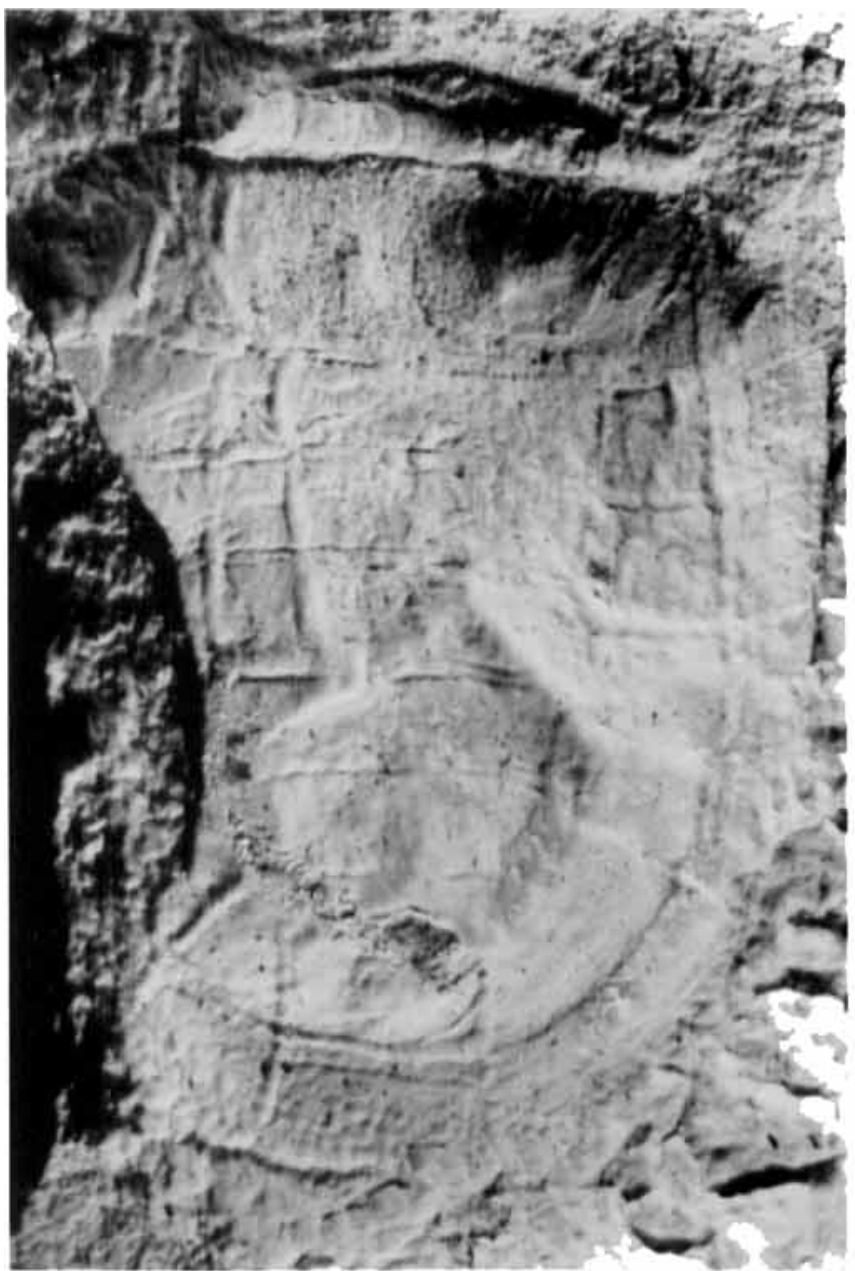

3
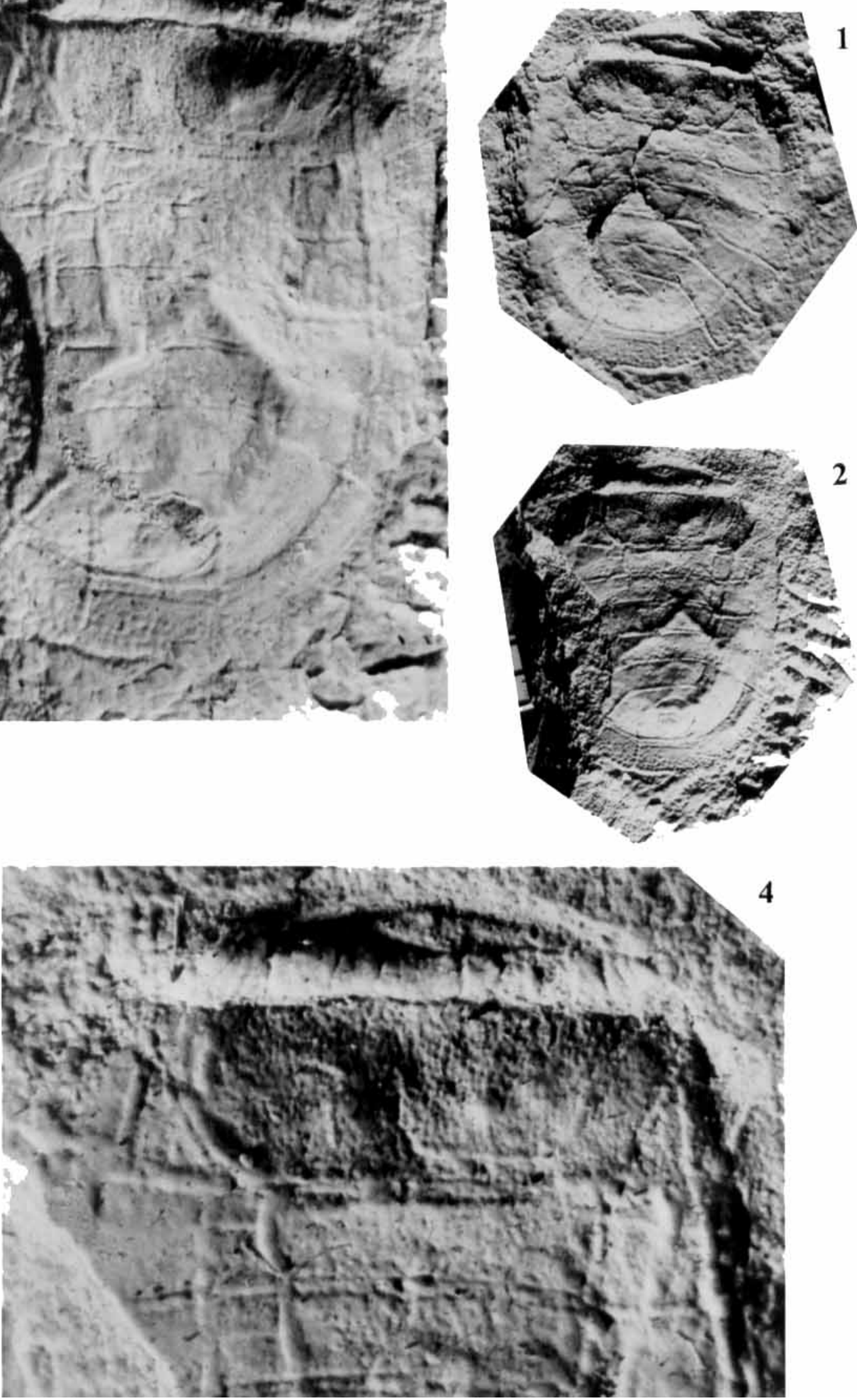
In der Arbeit von Nindel (1955) wurden u. a. ein von Sterzel (1918) als cf. Anthracomartus beschriebenes Spinnentier sowie zwei weitere fragmentarisch erhaltene Reste als Anthracomartus sp. abgebildet. Die Merkmalsbeschreibung von Nindel (1955) bezog sich in seiner Abb. 3 auf ein abdominales Dorsalschild bei dem Cephalothorax, Pedipalpi, Extremitäten und Operculum fehlen. Dasselbe gilt für die Darstellung in seiner Abb. 1. Jedoch sind hier wenigstens noch das Operculum und zwei Longitudinal-Linien ausgebildet. Bereits bei Sterzel (1918) gehen aus der Beschreibung dieses Fossilrestes Zweifel an einer eindeutigen "taxonomischen" Zuordnung hervor.

Alle drei Fossilreste sind unter Zugrundelegung der Diagnose von Karsch (1882) nicht als Anthracomartus sp. zu diagnostizieren. Rößler \& Schneider (1997) bilden erstmals den von Sterzel (1918) dargestellten tierischen Rest fotographisch ab. Ohne Zweifel sind jetzt Merkmale sichtbar geworden (graphisch idealisiert), die Vergleiche mit nahestehenden Formen zulassen. Diagnostisch sicher scheinen die Segmentgrenzen und die durch Längssuturen bedingten Felderungen in mediane und marginale Tergite sowie die diesen eigenen Skulptierungen. Auch sind Coxa, Trochanter, Femur, Patella und Beinpaare erkennbar. Werden die neuerkannten Merkmale von Umriß und Skulptur berücksichtigt, so wäre eine Ähnlichkeit mit Aphantomartus sp. möglich.

\section{Dank}

Die Fotos wurden von Frau Waltraud Harre, Museum für Naturkunde, angefertigt.

\section{Literatur}

Brauckmann, C. 1984. Eine neue Arachniden-Art aus dem Westfalium des Saargebietes (West-Deutschland). Dortmunder Beiträge Landeskunde, naturwissenschaftliche Mitteilungen 18: 95-103.
- 1987. Neue Arachniden-Funde (Scorpionida, Trigonotarbida) aus dem westdeutschen Unter-Devon. - Geologica et Palaeontologica 21: 73-85.

- 1987. Neue Arachniden (Ricinuleida, Trigonotarbida) aus dem Namurium B von Hagen-Vorhalle (Ober-Karbon; West-Deutschland). - Dortmunder Beiträge zur Landeskunde, naturwiss. Mitt. 21: 97-109.

Corda, A. J. C. 1835. Ueber den in der Steinkohlenformation bei Chomle gefundenen fossilen Scorpion. - Verhandlungen Gesellschaft Vaterländischen Museums Böhmen.

Daber, R. 1990. Arachnidenrest aus dem Westfal D von Zwickau-Oelsnitz. - Zeitschrift für geologische Wissenschaften 18 (7): 679-682.

- 1992. "Autochthone" Arachnidenreste aus dem Stefan von Plötz (Wettiner Schichten). - Zeitschrift für geologische Wissenschaften 20 (5/6): 455-459.

Dunlop, I. A. 1996. Systematics of the fossil arachnids. Revue Suisse de Zoologie, série 173-184.

Guthörl, P. 1934. Die Arthropoden aus dem Carbon und Perm des Saar-Nahe-Pfalz-Gebietes. - Abhandlungen der Preußischen Geologischen Landesanstalt NF, 164: 34-44.

Haase, E. 1890. Beiträge zur Kenntnis der fossilen Arachniden. - Zeitschrift der Deutschen geologischen Gesellschaft 4: 629-657.

Hennig, W. 1969. Die Stammesgeschichte der Insekten. Frankfurt am Main (Verlag von Waldemar Kramer) 436 S., 143 Abb.

Kahlert, E. 1975. Dic Unterkarbon-Flora von Delitzsch. Zeitschrift für geologische Wissenschaften 3: 907-925.

Lamarck, J. B. 1801. Système des Animaux sans Vertèbres, ou Tableau général des Classes, des Ordres et des Genres de ces Animaux. - Par J. B. Lamarck, 452 S.

Nindel, F. 1955. Die tierischen Reste aus dem Karbon von Karl-Marx-Stadt und Hainichen i. S. - Geologie 4 (7/8): 673-694.

Petrunkevitch, A. 1945. Palaeozoic Arachnida of Illinois. An inquiry into their evolutionary trends. - Sci. Pap. Lllinois 3 (2): $1-72$.

- 1949a. A Study of Palaeozoic Arachnida. - Academy Arts Science 37: 69-315.

- 1953. Paleozoic and Mesozoic Arachnida of Europe. The Geological Society of America 53: $1-128$.

Pocock, R. I. 1911. A monograph of the terrestrial carboniferous arachnida of Great britain. Printed for the Palaeontographical Society.

Rößler, R. \& Schneider, J. 1997. Eine bemerkenswerte Paläobiocoenose im Unterkarbon Mitteleuropas -- Fossilführung und Paläoenvironment der Hainichen-Subgruppe (Erzgebirgs-Becken). - Veröffentlichungen Museum für Naturkunde Chemnitz 20: 5-44.

Sterzel, J. T. 1918. Die organischen Reste des Kulms und Rotliegenden der Gegend von Chemnitz. - Abhandlungen der mathematisch-physischen Klasse der königlichen sächsischen Gesellschaft der Wissenschaften V: 209-210.

Simon, R. 1971. Neue Arthropodenfunde aus dem Stephan der Halleschen Mulde. - Berichte der Deutschen Gesellschaft für Geologische Wissenschaften, A Geologie und Paläontologie 16 (1): 53-62. 